\title{
O PAPEL DAS REDES SOCIAIS NO PROCESSO DE APRENDIZAGEM ORGANIZACIONAL: UM ESTUDO DE CASO EM SUCURSAL DE BANCO PORTUGUÊS
}

\author{
Augusto Antunes* \\ Mário Franco ${ }^{\dagger}$
}

\begin{abstract}
Resumo: $O$ objetivo deste trabalho é estudar a influência das redes sociais na aprendizagem organizacional. Um estudo qualitativo estudo de caso simples - foi utilizado para recolher dados através de entrevistas presenciais com os membros-chave, mais envolvidos numa sucursal de um Banco Português. Os dados foram analisados com uma abordagem qualitativa: análise de conteúdo. Os resultados enfatizam o impacto das redes sociais, a força dos laços e amizade na aprendizagem organizacional. A partir das evidências empíricas, podemos concluir que a administração e os diretores de topo a nível organizacional precisam prestar maior atenção às redes sociais em que ocorrem os esforços de transferência de conhecimento. Recursos trocados e desenvolvidos através de interações sociais devem ser concebidos para aumentar os fluxos de conhecimentos entre os colaboradores. Finalmente, este artigo associa os dois corpos de pesquisa, muitas vezes estudados separadamente na investigação organizacional: literatura em redes sociais e a aprendizagem organizacional. Espera-se que o trabalho contribua para uma fertilização cruzada dos dois campos.
\end{abstract}

Palavras-chave: redes sociais, aprendizagem organizacional, conhecimento, liderança, estudo de caso.

* Mestre em Gestão, Centro Regional das Beiras, Universidade Católica Portuguesa. e Doutorado em Gestão, Universidade da Beira Interior. E-mail: paisantunes@hotmail.com

${ }^{\dagger}$ Docente da Faculdade de Ciências Sociais e Humanas, Universidade da Beira Interior. E-mail: mfranco@ubi.pt 


\title{
THE ROLE OF SOCIAL NETWORKS IN ORGANIZATIONAL LEARNING: A CASE STUDY IN A PORTUGUESE BANK BRANCH
}

\begin{abstract}
The purpose of this paper is to study the influence of social networks on organizational learning. A qualitative study simple case study - was used to collect data, via face-to-face interviews with key members that are the most involved in a Portuguese Bank. Data were analyzed with a qualitative technique based: content analysis. Results emphasize the impact of social networks, strength of ties and friendship on organizational learning. From the empirical evidences, we conclude that top management and directors at an organizational level need to pay careful attention to the social networks within which knowledge transfer efforts occur. Resources exchanged developed through social interactions must be designed to increase knowledge flows between collaborators. Finally, the paper links two bodies of research often studied separately in organizational research: literature on social networks and that on organizational learning. It is hoped that the paper contributes to a cross-fertilization of the two fields.
\end{abstract}

Key words: Social networks, Organizational learning; knowledge, leadership, case study.

\section{INTRODUÇÃO}

As últimas décadas têm sido férteis em transformações económicas sem precedentes à escala global. Particularmente no sector financeiro, destaca-se a abertura dos mercados de capitais e a sua catastrófica desregulação, aliada aos grandes avanços tecnológicos dos sistemas de informação (Krugman, 2009). Como refere Davis (1990), a gestão dos bancos comerciais tem estado a passar por uma crise de desregulamentação, de competição muito intensa e de introdução de novas tecnologias e novos produtos. A este propósito, McKechnie (1992) e Devlin \& Ennew (1997) argumentam que, na organização financeira, não obstante haver características comuns à generalidade dos serviços, os serviços financeiros distinguem-se por algumas propriedades específicas: regime de trabalho intensivo e dispersão geográfica - prestação de serviços executada através de múltiplos locais: físicos e virtuais.

Nestas circunstâncias, pode dizer-se que as redes sociais, particularmente, as redes de confiança estabelecidas pelos gestores de topo devem ter como objetivo fulcral o desenvolvimento e a eficácia das suas organizações, de molde a serem verdadeiros exemplos para a aprendizagem organizacional. Para Shein (1993) e de forma a que as organizações consigam sobreviver, é necessário que aprendam a adaptar-- 
se mais rapidamente ou, então, acabarão por sair do processo de evolução económica. Tal como refere Senge (1996), o crescimento e desenvolvimento do mundo e a complexidade e dinamismo dos negócios propende a interligar-se com a própria aprendizagem. Aventamos que esta não acontece desligada nas organizações, mas sempre enquanto soma de várias componentes individuais - relacionamentos formais e informais, estabelecidos ao longo de várias interacções e que conduzem à eficácia e à vantagem competitiva propalada e baseada no conhecimento transferido de múltiplas formas.

De facto, alguns trabalhos realizados sobre o tema das redes sociais mostram que essas relações interpessoais são de grande importância para a aprendizagem organizacional. No entanto, nota-se que estes estudos assumem um impacto direto das redes sociais na transferência de conhecimento, pressupondo implicitamente que as redes sociais geram recursos que são úteis para a transferência de conhecimento. Consequentemente, os efeitos das redes sociais sobre a aprendizagem organizacional, são equiparados a uma "caixa negra" (Chollet, 2005) que nos propomos entreabrir neste estudo.

Neste sentido, a capacidade de uma organização pode entender-se como resultado das equipas de recursos trabalhando em conjunto. Ela pode ser identificada e avaliada através de um padrão de classificação funcional de atividades. Neste estudo, evidenciam-se as competências distintivas existentes em determinadas empresas. Para Barney (1991), Grant (1991) e Wernerfelt (1984) a criação de capacidades não é simplesmente uma questão de montar uma equipa de recursos já que ela envolve bases complexas de coordenação entre as pessoas e outros recursos. Assim, os recursos devem ser perfeitamente inimitáveis e não substituíveis pelos concorrentes.

Assim, tratando-se de um fenómeno atual, em profunda e acelerada mudança, o principal objetivo deste estudo é perceber adequadamente o papel das redes sociais na aprendizagem organizacional, particularmente, em contexto bancário real em Portugal.

Para tal propósito, permanecem algumas questões de investigação que, sendo respondidas, trarão benefícios ao entendimento desta relação em estudo: (1) Como se estabelecem relações de confiança na prática diária? (2) Qual a influência da amizade nas relações profissionais?; (3) Como se perceciona o impacto da liderança na organização?; (4) Como se processa o estabelecimento de laços?; (5) De que forma a partilha de informações contribui para a aprendizagem individual?; $e$ (6) De que forma o ambiente/clima profissional contribui para o desenvolvimento da organização/obtenção de resultados? 
A nível prático este estudo procura contribuir para uma visão do interior de uma organização financeira, apontando caminhos para a gestão de topo seguir ou alterar comportamentos enraizados, relações instituídas, em prol do desenvolvimento de processos de aprendizagem contínua. Para este desiderato, elaborou-se um estudo de caso no Banco X - Banco Português de referência, uma "organização orientada para a aprendizagem" para a "partilha de conhecimentos" e a "formação de colaboradores como prioridade indispensável à criação de valor". As mudanças repentinas e imprevisíveis no sector financeiro, em particular as derivadas da crise subprime de 2008, constituiram também leitmotiv para a melhor compreensão desta temática.

O restante deste artigo está organizado da seguinte forma. Na próxima seção, apresenta-se uma revisão da literatura sobre as redes sociais e aprendizagem organizacional - dois mecanismos através dos quais é passível uma boa transferência de conhecimento. Apresenta-se também um modelo de pesquisa. Na secção 3 , descreve-se o estudo de caso, o processo de recolha de dados e as variáveis selecionadas. Na secção 4, apresentam-se os resultados empíricos e a discussão dos resultados. Por fim, elabora-se a conclusão da investigação e são apresentadas algumas implicações teórico-práticas com base nestas conclusões. São aventadas algumas ideias para futuras investigações.

\section{ENQUADRAMENTO TEÓRICO}

\subsection{Redes Sociais}

As pessoas estão inseridas na sociedade por meio das relações que desenvolvem durante toda sua vida, primeiro no âmbito familiar, em seguida na escola, na comunidade em que vivem e no trabalho. As relações que as pessoas desenvolvem e mantêm são as que fortalecem a esfera social. A própria natureza humana liga-nos a outras pessoas e estrutura a sociedade em rede. Para Estevão \& Franco (2010), as redes são consideradas uma importante forma de criar oportunidades e transferir conhecimento, dado que a formação de extensas redes de relacionamentos possibilita, direta ou indiretamente, a maior perceção do meio envolvente. Castells (1999) faz uma relação direta das redes com a sociedade na Era da Informação e define-as como um conjunto de elos e nós interligados. Assim, as redes demonstram serem facilitadoras da aquisição de uma variedade de recursos organizacionais importantes para a atividade empreendedora (Rejeb-Khachlouf \& Mezghani, 2011).

Também Cunha et al. (2007) referem que as mudanças no mundo do trabalho e a criação de organizações com fronteiras cada vez mais fluidas, fizeram aumentar a importância das redes sociais. Para estes autores, a 
probabilidade de um profissional procurar informação ou conhecimento junto de um amigo ou colega é cinco vezes maior do que procurar essa informação numa fonte impessoal. Cross \& Sproull (2004: 451) argumentam que "as pessoas participam em diferentes redes para obterem distintos tipos de recursos: informação, validação e legitimação. As redes facilitarão a circulação mais rápida da informação no seio da organização, permitindo que ela esteja disponível onde é necessária. As redes permitem ainda sinergias que transformam informação episódica em conhecimento e em recursos para a ação".

Seguidamente são descritas algumas variáveis e/ou dimensões que estão associadas às redes sociais.

\section{i) Confiança}

Relativamente à gestão da confiança, Handy (1995) refere que, especialmente na era das organizações virtuais, não se trata de um conceito fácil de operacionalizar pelo que aponta para a necessidade de estabelecimento de sete regras básicas: (1) a confiança não é cega (tem que existir um tempo para o conhecimento das pessoas); (2) a confiança tem necessidade de limites (avaliação pelos resultados); (3) a confiança necessita de aprendizagem; (4) a confiança não admite perdão; (5) a instituição de múltiplos tipos de ligações; (6) a confiança exige líderes que aceitem a partilha da liderança; e (7) a confiança implica necessidades de contacto intenso para levar ao sentimento de pertença.

$\mathrm{O}$ intercâmbio de ideias, opiniões e crenças propiciado pelas conversas, possibilitam o primeiro e o mais importante passo para a criação do conhecimento: a partilha do conhecimento tácito dentro da comunidade da rede. Como referem Berliant \& Fujita (2009), à medida que as pessoas criam e transferem conhecimento, este vai-se alterando.

Para Imai (1989), uma rede é na sua essência um período de influências que existe num determinado sistema e num determinado espaço, como resultado de um processo dinâmico de acumulação de experiências e aprendizagem, isto é, uma estrutura em constante mudança. Assim, para Gulati (1998), as redes sociais das organizações são determinantes na seleção do parceiro para a cooperação e a interdependência de recursos é uma condição necessária, mas não suficiente, para que se estabeleçam relações de colaboração.

\section{ii) Liderança}

De acordo com Tichy \& Bennis (2010) a rede pode servir também para determinar o valor das relações que se estabelecem entre os indivíduos na qual a liderança tem um papel determinante no jogo da 
equipa, no seu alinhamento e nas relações que se estabelecem na organização entre todos os stakeholders.

O líder serve a rede como um motor de aproveitamento das capacidades, encorajando o trabalho em equipa e a utilização dos melhores recursos dos seus membros. Para os autores, "é $d a$ responsabilidade do líder construir processos de criação de conhecimento para a sua equipa e assegurar que estes processos são executados (p.273)". No fundo, trata-se do desenvolvimento de relações profissionais íntimas entre a liderança e os subordinados para que o processo de transmissão de informações e conhecimento seja fluído e aberto. Também resulta desta constatação que todos os líderes se apoiam num grupo de conselheiros em que confiam e a construção de uma rede social que desenvolve continuamente a capacidade de criação de conhecimento é um elemento central para o sucesso de um líder.

\section{iii) Amizade}

Para Rank \& Tuschke (2010), os gestores, na exigência quotidiana de cumprirem as suas funções complexas, procuram colegas em todos os níveis hierárquicos a quem possam prestar determinados benefícios, tais como: aconselhamento, informação e apoio. Em concreto, argumenta-se que a influência percebida, bem como os laços de amizade entre dois gestores aumentará a probabilidade de eles colaborarem entre si.

Stevenson \& Radin (2009) demonstraram que existe uma relação positiva entre o número de interações que um diretor tem com outros membros do Conselho de Administração e a sua influência sobre as decisões desse órgão. Westphal (1999) e McDonald \& Westphal (2003) investigaram o efeito dos $\mathrm{CEOs}^{\ddagger}$ na obtenção de pareceres de diretores de fora da organização, em temáticas ligadas a processos comportamentais e também no desempenho da empresa. Deste modo, argumenta-se que os gestores não só terão as suas características individuais ao decidirem com quem cooperar, mas também as suas decisões colaborativas serão orientadas pela forma como eles percebem os potenciais parceiros de cooperação.

$\mathrm{Na}$ literatura de redes sociais, a amizade assumiu ter efeitos positivos sobre a inserção dos intervenientes em redes de aconselhamento, especialmente quando existe incerteza nos membros organizacionais (Krackhardt, 1990, Krackhardt \& Stern, 1988, citados em Rank \& Tuschke, 2010). Da mesma forma Uzzi (1996) enfatizou os efeitos positivos que os laços de amizade podem ter na resolução conjunta de problemas.

\footnotetext{
${ }^{\ddagger}$ Chief Executive Officer - Diretor Executivo
} 
Strauss (1962) chegou a propor que confiar nas amizades pode ser considerada como uma táctica de poder. Este autor observou que os trabalhadores que têm de cooperar com frequência seguem o princípio de "recompensar os seus amigos, punir vossos inimigos " (p. 74), havendo assim uma rede de troca de favores.

O recurso relevante numa rede de amizade é o afeto ou a relação social. Os laços de amizade são, portanto, definidos como relacionamentos expressivos. Além do valor intrínseco de serem integrados numa rede de amizade, esta tem sido sugerida como instrumental para a obtenção de outros recursos relevantes, tais como informações importantes e também pode ser a base para a formação de coligações. Para reforço destas posições, a informação e conhecimento que são trocados entre amigos são mais credíveis e mais acessíveis (Brass 1992, Uzzi, 1996).

\section{iv) Estabelecimento de laços}

Segundo Håkansson e Johanson (1988), a cooperação é um elemento primordial em todas as estratégias e redes cooperativas. Birley (1985) descobriu que os empresários e gestores se relacionam extensivamente em redes informais de famílias, amigos e pessoas de negócios e menos em redes formais. Para Johannisson (1987), algumas destas redes pessoais são formadas por laços de amizade $a d$ hoc e são baseadas na confiança. Este tipo de redes parece vital para perceber oportunidades (Aldrich \& Zimmer, 1986). O acesso a inputs e a venda de outputs requerem que as empresas estabeleçam ligações diretas e indiretas não só com clientes e fornecedores, mas também com concorrentes, clientes de clientes, fornecedores complementares, ou seja a interdependência evidente entre eles (Johanson \& Vahlne, 1990; Roseira, Brito \& Henneberg, 2010).

Holm et al. (1995) referem que as organizações são mais capazes de perceber oportunidades em direção a uma rede de empresas, bem como praticar elas próprias a colaboração, quando estas organizações são capazes de mobilizar relacionamentos dentro dessa rede. Para Johanson \& Vahlne (1990), estes relacionamentos estão conectados por redes que se desenvolvem como uma consequência da interação entre as empresas.

Hansen (1999) prova que laços fortes garantem a transferência de conhecimentos complexos, ao passo que os laços fracos são mais eficazes para a transferência de conhecimentos simples. Assim, a aprendizagem não é apenas localizada nos esquemas mentais e nas experiências anteriores dos trabalhadores, mas também nas relações que mantêm com os atores externos (Uzzi \& Lancaster, 2003). 


\subsection{Aprendizagem Organizacional}

De acordo com Borgatti \& Cross (2003), um corpo significativo e crescente da literatura tem vindo a abordar a aprendizagem organizacional e a sua evolução tem-se acentuado ao longo dos últimos 30 anos. Todavia, para Kim (1993), nos anos 90, a teoria sobre a aprendizagem organizacional estava ainda num estado embrionário. Também para Franco \& Ferreira (2007: 171), “a literatura revela ainda a falta de um corpo teórico convergente sobre as organizações que aprendem... Este resultado surge possivelmente do carácter multidisciplinar nesta área do conhecimento."

De acordo com Lopes \& Fernandes (2002), a preocupação com a aprendizagem organizacional passou, a partir dos ano 90 , a ter um grande destaque no domínio das ciências das organizações. Assim, as teorias desenvolvidas sobre a temática têm partido dos modelos de aprendizagem individual (Dodgson,1993; Popper \& Lipschitz, 2000) o que tem feito com que a investigação neste domínio não evolua tanto como seria desejável para se compreender a realidade organizacional.

Para Senge (1990), a aprendizagem organizacional é um processo pelo qual uma organização se torna inteligente, porque aprende e expande continuamente a sua capacidade para criar o futuro. As organizações inteligentes são capazes de se sobreporem às dificuldades, de reconhecerem as ameaças e de enfrentarem as oportunidades. Já Cabrita (2009) e Garvin (1993) definem uma organização que aprende como uma organização especializada, um processo dinâmico, em criar, adquirir e transferir conhecimento e em modificar a sua acção para reflectir os novos conhecimentos que garantem a sustentabilidade das suas vantagens competitivas.

Popper \& Lipschitz (2000) sugerem que apesar da aprendizagem individual e organizacional envolverem o processamento de informação, necessitam de diferentes mecanismos para converter as informações para o conhecimento em diferentes níveis sistémicos: a aprendizagem organizacional produtiva requer uma cultura de aprendizagem que consiste num compromisso com a transparência.

Também no âmbito da aprendizagem organizacional se pode descrever algumas dimensões/variáveis que tornam este tipo de processo organizacional eficaz. Seguidamente são descritas algumas destas dimensões.

\section{i) Níveis e Programas de Aprendizagem}

Argumenta-se que existem três níveis principais de aprendizagem: (1) individual, (2) do grupo e (3) da organização. Unger \& Lorscheider 
(1996) consideram os grupos como elos fundamentais entre a aprendizagem individual e organizacional. Crossan et al. (1999), por sua vez, mostram que a aprendizagem organizacional é um processo dinâmico que implica movimento entre os diferentes níveis de atuação, passando a partir do nível individual ao nível do grupo, deste para o nível organizacional e também no sentido inverso.

$\mathrm{Na}$ visão de Senge (1990), as organizações devem desenvolver programas permanentes de estudo e prática necessários à aprendizagem organizacional. Esta está relacionada com os grandes desafios das organizações e as pessoas aprendem mais e de forma mais rápida quando estão comprometidas com a sua missão, pelo que estas cinco disciplinas são baseadas em capacidades fundamentais: (1) Dominar e comunicar conceitos; (2) Criação de modelos mentais; (3) Partilhar uma visão de futuro; (4) Aprendizagem em equipa; 5) Capacidade de pensamento sistémico.

\section{ii) Aprendizagem individual}

Alguns autores (Crossan et al., 1999; Popper \& Lipshitz, 2000) compreendem as organizações que aprendem como a aprendizagem individual que ocorre no contexto organizacional, uma vez que é o indivíduo que possui a capacidade de agir nesse contexto. Uma abordagem ligeiramente diferente sugere que a aprendizagem individual é um pré-requisito para a aprendizagem organizacional (Hedgerg, 1981; Kim, 1993; Dogson, 1993; Senge, 1996). Dogson (1993: 377), por exemplo, sugere que a aprendizagem organizacional é o resultado da aprendizagem individual e que "os indivíduos são as entidades primárias de aprendizagem nas organizações e são os indivíduos que criam formas organizacionais que possibilitam a aprendizagem de tal forma que facilite a transformação organizacional".

Cohen \& Levintal (1990) apresentam uma posição similar ao afirmarem que as estruturas cognitivas de cada indivíduo numa organização proporcionam a base para a aprendizagem organizacional. Nonaka \& Takeuchi (1997) também indicam que o conhecimento só pode ser criado por indivíduos e que a aprendizagem individual é a base para a compreensão do processo de aprendizagem organizacional. Dogson (1993) reconhece a necessidade da aprendizagem individual como necessária para a aprendizagem organizacional, mas reforçam que a aprendizagem organizacional significa mais do que a soma agregada das aprendizagens individuais. Segundo Cohen (1998), considerando-se essa realidade, o principal desafio dos profissionais da área de Gestão de Recursos Humanos, executivos e outros profissionais do conhecimento é 
o de criar estruturas para auxiliar na conversão do conhecimento individual em conhecimento organizacional.

A incorporação do conhecimento individual no capital de conhecimento da organização baseia-se no facto de que as organizações podem ser representadas por padrões de interações entre indivíduos que tendem a perdurar mesmo quando alguns desses indivíduos deixam a organização (Inkpen, 1996; Inkpen \& Crossan, 1995).

\section{iii) Transferência de Conhecimento}

É com base nos conhecimentos tácitos e explícitos que os indivíduos poderão adquirir novos conhecimentos, bem como transmitir conhecimentos já possuídos. Estes fenómenos são-nos apresentados por Nonaka \& Takeuchi (1995), através da sua denominada espiral do conhecimento.

Kogut \& Zander (1996) relevam que a empresa/organização deve ser entendida como uma comunidade social especializada na rapidez e eficiência na criação e transferência de conhecimento. Da sua perspectiva resulta que a vantagem das organizações tem uma base estabelecida a partir de relações sociais que se estabelecem entre os seus membros e que conduzem à transmissão de vários tipos de conhecimento.

Neste contexto, a aprendizagem organizacional aparece como um processo em construção, baseando-se o seu estudo preferencialmente nos modelos de aprendizagem individuais. Entretanto, a necessidade de separar a aprendizagem organizacional da individual tem vindo a imporse. A aprendizagem organizacional ocorre, necessariamente, através dos indivíduos, mas não se resume ao somatório de conhecimentos dos diversos indivíduos - é um fenómeno mais complexo e dinâmico que resulta da interação entre os indivíduos. Também não é um prolongamento das aprendizagens dos gestores, pois existem organizações em que os gestores mudam e as organizações permanecem idênticas (Lopes, 2008).

\section{iv) Clima organizacional}

Billett (2004) descreve a aprendizagem no local de trabalho, em termos de participação do aluno/aprendiz em atividades de trabalho e enfatiza práticas de engajamento e participação dos indivíduos. O conceito de "aprendizagem e ambiente de trabalho" é derivado de pesquisas anteriores (Birt et al, 2004; Visser, 2001). Em geral, refere-se ao ambiente onde os funcionários aprendem e trabalham. Mais especificamente, o conceito poderia ser descrito, referindo-se: orientação e valorização no trabalho, pressão de trabalho, a quantidade de autonomia e de responsabilidade que os funcionários comportam; escolha de tarefas 
de trabalho e desenvolvimento; prestação de trabalho desafiador e significativo e oportunidades de desenvolvimento.

Kholer \& Jansen (2010) num artigo sobre os determinantes do clima de inovação em bancos germânicos, referem que o clima organizacional desempenha um papel significativo na implementação de inovações. Os fatores que afetam este clima são as perceções socialmente compartilhadas, práticas organizacionais, procedimentos e valores. Exemplos notáveis dessas perceções compartilhadas, incluem o foco de uma organização em objetivos comuns, os esforços feitos por membros da equipa para alcançar um elevado nível de desempenho, a cooperação de membros da equipe e o apoio mútuo para o desenvolvimento e aplicação de novas ideias (King e Anderson 1995).

\subsection{Proposta de Modelo Conceptual de Análise}

Com base na revisão da literatura existente, propõe-se um modelo conceptual onde são estabelecidas várias dimensões que albergarão o conjunto relevante dos principais temas inter-relacionados: as redes sociais e a aprendizagem organizacional (Figura 1). Procura-se assim, estabelecer uma síntese que procure demonstrar a realidade em estudo. Todavia, para Franco \& Ferreira (2007:178), “um modelo, é por definição, uma simplificação de uma realidade complexa e existe sempre a possibilidade de incluir, ou não, certas variáveis e dimensões no mesmo".

\section{Figura 1}

Modelo conceptual de análise

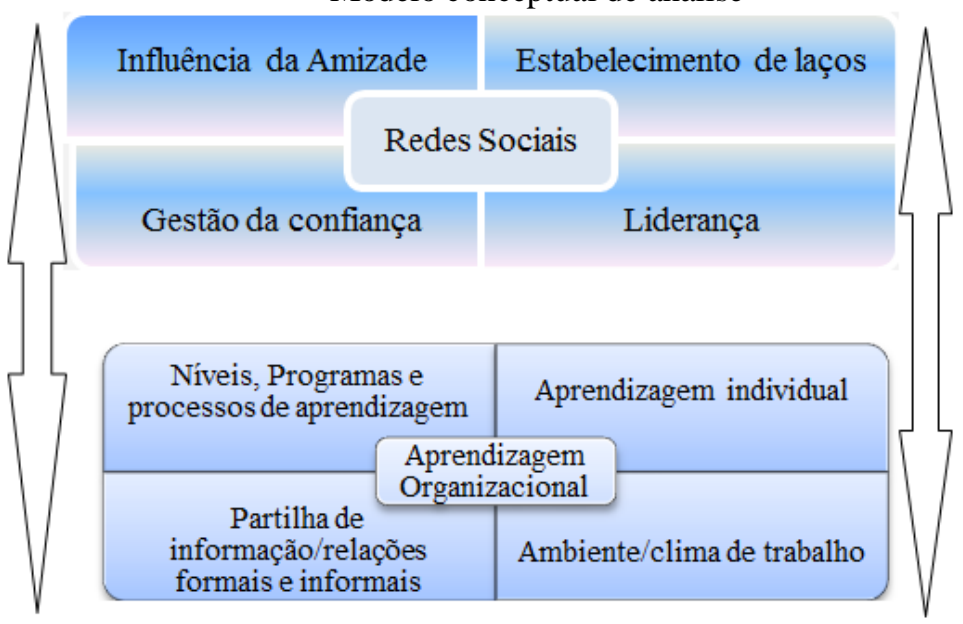

Fonte: construção própria 
Esta ilustração procura elucidar sobre as oito dimensões em estudo: redes sociais $(\mathrm{RS})$ = Influência da amizade, estabelecimento de laços, gestão da confiança, liderança e Aprendizagem Organizacional $(\mathrm{AO})=$ Níveis, programas e processos de aprendizagem, partilha de informação/relações formais e informais, aprendizagem individual e ambiente/clima de trabalho.

\section{METODOLOGIA DE INVESTIGAÇÃO}

\subsection{Tipo de estudo}

Optou-se por uma abordagem de pesquisa qualitativa dada a natureza social do fenómeno. Trata-se aqui de um contexto objetivo, natural e contemporâneo. Dentro desta abordagem de investigação decidiu-se ainda pela estratégia de estudo de caso. Para Yin (2005) e Sjoberg et al. (1991), um estudo de caso examina um fenómeno através de abordagem holística no seu contexto natural, empregando múltiplos métodos para recolher informação de uma ou de poucas entidades (pessoas, grupos ou organizações).

\subsection{Seleção do Caso}

Nas perspetivas de Bonoma (1985), Easterby-Smith et al. (2010), Eisenhardt (1989), Mills et al. (2010) e Yin (2005), o estudo de caso envolve a análise intensiva de um número relativamente pequeno de situações e, às vezes, o número de casos estudados reduz-se a um. É dada ênfase à completa descrição e ao entendimento do relacionamento dos fatores de cada situação, não importando os números envolvidos.

Para a validação do estudo de caso aqui selecionado, utilizaram-se as estratégias propostas por Yin (2005). Apresenta-se um único caso Banco X, pelo que a unidade de análise é esta organização.

Para a seleção do Banco X, utilizaram-se os seguintes critérios:

- Escolheu-se o Banco X por razões de conveniência: facilidade de acesso à informação;

- Existência de práticas de aprendizagem individual e organizacional de reconhecido interesse;

- Recolha de informação pelo investigador em contexto real;

- Organização integrada em sector dinâmico, em mudança permanente e onde o conhecimento e a experiência têm um relevante papel;

- Confiança - argumento base deste tipo de organizações. 


\subsection{Recolha de Informação e Análise}

Os métodos e técnicas de recolha de dados foram as entrevistas, a observação participante e direta necessárias à triangulação de dados (Scandura \& Williams, 2000; Yin, 2005).

O protocolo da entrevista, não estruturada e informal, foi baseado na literatura analisada (Coutinho, 2011; Eisenhardt, 1989; Quivy \& Campenhoudt, 2008; Yin, 2005) e nas dimensões do modelo conceptual proposto. As entrevistas tiveram lugar em Maio de 2013 e, em média, duraram entre 45-60 minutos. Foram também analisados outros documentos, e que, por razões de confidencialidade, não são explanados neste estudo.

As entrevistas foram efetuadas a quatro colaboradores: (1) Sílvia Santos (SS), gestora de relações comerciais, possuidora de 22 anos de experiência bancária e detentora de uma licenciatura em Economia; (2) Luís Cunha (LC), gestor de operações, tem 14 anos de Banca e é detentor de uma pós-graduação em Gestão; (3) Sandra Ferreira (SF), gestora de clientes, há 18 anos na empresa e detentora de uma licenciatura em Economia; e (4) Ricardo Rodrigues (RR), diretor de PMEs, 12 anos de banco, licenciado em Gestão.

As entrevistas foram tratadas através da ferramenta informática NVivo 9.2 e tiveram como base aquilo que os entrevistados explanaram, com posterior validação. Foi adotada a análise de conteúdo como técnica de análise.

\section{ESTUDO DE CASO}

\subsection{Caracterização do Banco X}

O Banco X é integrante do índice PSI 20 com mais de 7000 trabalhadores a nível global. Possui sucursais ou instituições em países da África, Europa e Ásia. É notada empresa socialmente responsável.

Possui como idade média dos seus colaboradores os 42 anos e com uma elevada percentagem de colaboradores com habilitações superiores ao $12^{\circ}$ ano. Nas funções técnicas e de contacto com clientes (ex. empresas) a predominância é para os empregados possuidores de cursos superiores ao nível da Economia e Gestão, com elevada formação básica em produtos, serviços e aconselhamento financeiro proporcionados pelo Banco. $\mathrm{Na}$ opinião do seu CEO, existe uma preocupação interna em relação às mudanças atuais e uma clara orientação para os novos desafios e comportamentos exigíveis pela sua base de clientes. 
Tendo presente, o tipo de atividade e a exigência de regulamentos legais que a enquadram, a estrutura organizacional é conservadora, verticalizada e a comunicação da informação é efetuada através de vários canais físicos e tecnológicos.

\subsection{Resultados e Discussão}

A interpretação da informação processada foi organizada com base nas seis dimensões do modelo (Figura 1) e aqui explanada por extratos relevantes conforme inquirição individual e reduzida ao seu conteúdo básico por razões de tempo e eficácia.

\subsubsection{Redes Sociais}

\section{i) Confiança}

De acordo com Cross, Parker \& Prusak (2000), a criação de um maior grau de segurança dentro das relações organizacionais, permite um aumento da aprendizagem e criatividade que são dependentes dos relacionamentos e que se desenvolvem ao longo do tempo. Davenport \& Prusak (1998) realçam algumas barreiras para a transferência do conhecimento nas organizações. Dentro destas, algumas podem ocorrer também nas redes sociais, como é o caso da falta de confiança mútua, diferentes culturas e quadros de referência distintos. Para estes autores são barreiras passíveis de serem facilmente superadas, dadas as características específicas das redes sociais.

Neste seguimento, para Luís Cunha, o Banco X aqui estudado tem estimulado um programa de encontros para incentivar e reforçar a confiança mútua. Outras organizações têm incentivado o contacto pessoal, através de reuniões mensais entre diferentes grupos de colaboradores, permitindo estimular uma atmosfera de confiança e cooperação entre todos os seus funcionários. Como refere este entrevistado:

"A confiança no trabalho das pessoas é determinante: tranquiliza quem lidera e faz crescer quem executa, procurando sempre fazer melhor e descobrir novas formas para superar objetivos, fazendo com que no limite a organização evolua".

Um outro dos entrevistados, Silvia Santos, refere também que, "por outro lado, a experiência e convivência de vários anos, cria laços de confiança que permitem agilizar alguns procedimentos...".

Ainda sobre a confiança necessária dentro das redes sociais, Sandra Ferreira sublinhou que: 
"Procuramos mais rapidamente um conselho em caso de dúvida em alguém em quem confiamos e isso resolve muitas situações mais complicadas".

\section{ii) Amizade}

$\mathrm{Na}$ literatura de redes sociais, a amizade assume ter efeitos positivos sobre a inserção dos intervenientes em redes de aconselhamento, especialmente quando existe incerteza nos membros organizacionais (Rank \& Tuschke, 2010). Aliás, Uzzi (1996) enfatizou os efeitos positivos que os laços de amizade podem ter na resolução conjunta de problemas.

Neste contexto, Luís Cunha pensa "que as ligações pessoais facilitam, em muito, a resolução de problemas, mesmo numa empresa eficiente como a nossa com parâmetros e níveis de serviço bem definidos $e$ organização superior".

Para SS, "por exemplo é provável que um colega que conhecemos pessoalmente não se limite a devolver um processo de crédito porque não está em conformidade, mas que telefona e permite que a situação seja resolvida com uma informação adicional".

Ricardo Rodrigues partilha a seguinte opinião:

"Efetivamente a construção de relações de proximidade entre os colaboradores reverte a favor de uma maior eficácia empresarial, uma vez que ultrapassadas as barreiras de origem primária (medo original), os colaboradores ficam em posição de darem o seu melhor. ... Como via de criar futuras empresas mais competitivas, recorrer a uma politica de estimulo do aprofundamento das relações interpessoais das várias estruturas empresariais...".

A amizade tem sido considerada em vários estudos de redes organizacionais (e.g., Brass, 1984; Kilduff, 1992; Kilduff \& Krackhardt, 1994; Mehra, Kilduff \& Brass 2001; Rank \& Tuschke, 2010) como uma dimensão que é definida como vínculos pessoais entre os gestores e que consiste em atividades conjuntas que não estão relacionadas com as suas atribuições normais relacionadas com o trabalho, i.e., a socialização fora do trabalho, os convites e visitas de casa. A amizade aumenta os comportamentos de procura de aconselhamento dos gestores (Westphal, 1999), como consequência, os laços de amizade entre os executivos podem promover a eficácia do processo de formulação deestratégias.

\section{iii) Liderança}

Para Rank \& Tuschke (2010), os altos executivos são quase sempre necessariamente poderosos e influentes. Da sua posição formal, espera-se 
diferenças entre os escalões superiores em relação às suas posições informais dentro da rede, bem como o seu acesso a recursos escassos.

Como referem Tichy (1997), Charan \& Tichy (1998) e Davis \& Useem (2001), os gestores de topo moldam a organização e orientam-na, são os altos executivos que ilustram a visão e mobilizam as fileiras. Para estes autores, os gestores de topo das organizações são vistos como uma certa personificação do capitalismo que, sendo impessoal, reveste-se de uma face personificada em 5,7,9, 11 senhores que orientam as grandes empresas e executam as estratégias definidas.

Neste seguimento, para o entrevistado (RR), "uma organização pode e deve ser caracterizada por ter objetivos, missão e cultura comuns a todos os participantes. É isso que está no ADN da nossa empresa e que nos é transmitido na primeira reunião de acolhimento ou de integração no Banco".

Também SS refere "a importância da liderança forte, motivadora, positiva que é necessária para o bom desempenho na sua sucursal e que organiza sessões out-of-box para as pessoas conseguirem dar ênfase ao importante. Nós sentimos que a nossa hierarquia ajuda-nos a fazer deste banco a Escola da Banca em Portugal e temos orgulho em pertencer a este projeto".

\section{iv) Estabelecimento de Laços}

Como referem Davis \& Useem (2001), existe uma série de estudos que confirmam o que muitos executivos já tinham notado: o melhor preditor de desempenho de uma organização, para além da capacidade do líder e sua capacidade de execução, é a qualidade da equipa e as interrelações que se estabelecem.

Numa instituição financeira, em que a experiência é fundamental, o estabelecimento de laços nas equipas é basilar na organização. Deste modo, para SF:

"O conhecimento de anos pode influenciar decisões/ análises/ aprendizagem sob diversas perspetivas, Por exemplo, numa situação de dúvida relativamente a uma tomada de decisão, conhecer-se o trabalho e a "forma de estar" de quem está a apresentar a questão, poderá ser decisivo nessa mesma decisão...”.

Também de acordo com LC, "muitos dos produtos e serviços nascem da relação estreita entre os diferentes departamentos. $O$ gestor começa a identificar uma(s) determinada(s) necessidades do(s) cliente(s), se não reportar com fidelidade o sentimento identificado, dificilmente os serviços identificarão a oportunidade de negócio. Para além disso, as relações de trabalho e informais facilitam a troca de conhecimento a nível das práticas de sucesso ou de insucesso, permitindo à organização 
ir corrigindo ao longo do tempo, de modo a satisfazer o cliente e alcançar padrões de excelência."

\subsubsection{Aprendizagem Organizacional}

\section{i) Niveis e Programas de Aprendizagem}

De acordo com Kim (1993: 2), "o dicionário aponta para a sua definição que é a aquisição de conhecimento ou capacidade/ habilidade/ competência. Assim, a aprendizagem comporta duas formas: (i) aquisição da capacidade ou hnow-how que implica uma habilidade física para produzir determinada acção, e (ii) a aquisição do "saber porquê" que implica uma capacidade para articular a compreensão conceptual de uma experiência".

$\mathrm{Na}$ perspetiva de Cascio (1995) estão-se a desenvolver alterações dramáticas no mundo do trabalho com a globalização imparável, o impacto das tecnologias e a reengenharia de processos. É assim que se assiste à mudança de estruturas verticais para dar lugar a redes de especialistas (Mintzberg, 2010).

A partir do testemunho recolhido junto do entrevistado RR, enquanto responsável de equipa:

- "É bom saber qual a direção que é apontada quando temos que cumprir determinados objetivos. Também a brusca alteração de rumo sem a devida explicação, faz-nos sentir um pouco fora da organização, como um barco à deriva". Quando o sistema de gestão de pessoas nos envia para um curso de formação com a explicação prévia do que se espera da minha atuação, obriga-nos a ser melhor responsáveis no dia-adia e isso é transferido informalmente".

\section{ii) Aprendizagem Individual}

De acordo com Kim (1993) e Lopes \& Fernandes (2002), nos estádios iniciais da existência das organizações, a aprendizagem organizacional é sinónimo de aprendizagem individual, dado que a organização consiste num pequeno grupo de pessoas e numa estrutura mínima. À medida que a organização cresce, a distinção entre a aprendizagem organizacional e a aprendizagem individual emerge e vem ao de cima este sistema. Para haver aprendizagem organizacional tem de haver aprendizagem individual, dado que as organizações só conseguem aprender através da experiência e acções dos indivíduos.

Na perspectiva Nonaka \& Takeuchi (1995) e Kogut \& Zander (1995), a criação do conhecimento nas organizações assenta na dinâmica imposta à sua codificação, i.e., à forma como ela torna acessível o conhecimento 
aos que dele carecem. Assim, o conhecimento é transformado num código de forma a tornar-se organizado, explícito e de fácil compreensão.

Para RR, "no Banco X a aprendizagem individual é estratégica e é levada muito a sério, enquadrando uma vantagem da organização...No último ano, estive envolvido num plano anual de coaching via empresa externa e fizeram-me ver perspetivas que eu não tinha consciência que elas afetavam o meu desempenho individual e potenciou-as de molde a tornar-me um melhor líder... A organização preocupou-se com a minha aprendizagem e descoberta e isso vai-se refletir nela... é quase um boomerang".

\section{iii) Partilha de Conhecimentos/Experiências}

De acordo com Inkpen \& Tsang (2005), a transferência de conhecimento é o processo através do qual um membro de uma rede é afetado pela experiência do outro. A transferência de conhecimento manifesta-se através de alterações no conhecimento ou desempenho do destinatário.

Franco \& Barbeira (2009) referem que as redes podem ser entendidas como um mecanismo de aprendizagem, transferência e de partilha de conhecimentos dentro e entre organizações, bem como forma de controlar este importante recurso que é o conhecimento. Reiteram Nahapiet \& Ghoshal (1998: 242) "que as capacidades particulares das organizações para criarem e partilharem conhecimento deriva de um conjunto de factores, incluindo a facilidade especial das organizações de procederem à transferência de conhecimento tácito". Para Davenport \& Prusak (1998: 88), "a mais óbvia forma de transferir conhecimento é através da contratatação de gente inteligente e deixá-los dialogar uns com os outros ... a transferência de conhecimento nas organizações é permanente e espontânea".

No caso aqui estudado, SF refere que "entre pessoas das diversas áreas, ainda que não se conheçam pessoalmente, ao longo do tempo vão trabalhando em conjunto (Ex: direções de crédito/direções de operações), criando empatia e "à vontade" para se tirarem dúvidas, para questionar novos procedimentos desconhecidos para uma das partes, sendo veículo de aprendizagem... a troca de experiencias e de informação é mais fácil entre colegas que se conhecem há vários anos..."

Para Rego et al. (2007) a partir de diversas investigações analisadas, resultam convergências em que os colaboradores das organizações sentem a organização como sua pertença, exprimem valores de grupo, de alinhamento e missão, consideram o seu trabalho com significado, útil à sociedade, experimentam alegria no trabalho e percecionam que a organização lhes proporciona oportunidades de crescimento, daí a partilha de conhecimentos entre os membros que contribui para a sua evolução. 


\section{iv) Clima Organizacional}

Para Kholer \& Jansen (2010), a fim de promover um clima amistoso, também ao nível da inovação, as organizações devem estabelecer valores partilhados. Uma etapa ativa nessa direção envolve o reforço do capital social e de um sadio ambiente de comunicação através de decisões de gestão confiável. Vera \& Crossan (2004) referem que, quando existe um ambiente turbulento e de incerteza, um clima contextual de crise, ansiedade e de alto risco, as organizações procuram lideranças carismáticas que têm por objetivo ajudar a que os colaboradores entendam o ambiente como fonte de oportunidade, abertura, eficácia, autoestima e comprometimento com a organização e seus processos de aprendizagem.

Nestas circunstâncias, SF sublinhou que:

"Empiricamente posso constatar que ao longo de quase 20 anos de funções na banca, o clima/ambiente existente é de boa disposição e com boas condições de trabalho, induz ao sucesso e maior motivação e inovação, consequentemente, com resultados de vulto para a organização. Penso que na minha sucursal esse ambiente é fulcral para me sentir bem, envolvida e motivada com abertura de espírito para fazer de cada dia, um dia melhor na profissão que escolhi".

Um outro dos entrevistados, SS, realça também que:

"Adoro trabalhar aqui, especialmente quando existe humor, risos e vontade de colaborar de toda a equipa. Um espírito de entreajuda e descontraído faz com que até o cliente note que somos diferentes. Também quando exultamos com as vitórias, por mais pequenas que sejam, ajuda a manter-se um bom ambiente $e$ isso traduz-se nos resultados diários".

\subsubsection{Síntese e Principais Variáveis no Modelo}

O papel das redes sociais no processo de aprendizagem organizacional deve ser entendido como fulcral e de grande impacto ao nível do desempenho dos seus membros e globalmente pela organização. $\mathrm{O}$ conhecimento e aprendizagem individual sairão reforçados e, em consequência, globalmente a aprendizagem organizacional se as organizações atuarem com base nas dimensões aqui explanadas pelos entrevistados/responsáveis do Banco $\mathrm{X}$. $\mathrm{O}$ seu acolhimento trará vantagens competitivas às organizações que façam desta relação biunívoca o seu "modus vivendi". Afinal, e como referem Nahapiet \& Ghoshal (1998: 252), "a proposição fundamental da teoria sobre o capital social é que o estabelecimento de redes constitui uma valiosa 
fonte de benefícios de informação, i.e., "quem conheces" afecta "o que conheces", sendo as redes sociais uma forma de acesso aos recursos".

\section{CONCLUSÕES E IMPLICAÇÕES TEÓRICO-PRÁTICAS}

O objetivo deste estudo foi examinar o papel das redes sociais no processo de aprendizagem organizacional, tendo por base um estudo de caso numa sucursal bancária. Assim, face às evidências empíricas obtidas, parece-nos que esta organização deverá estar permanentemente atenta às dimensões analisadas: (1) Redes Sociais - Influência da amizade, estabelecimento de laços, gestão da confiança, liderança, e (2) Aprendizagem Organizacional - Níveis, programas e processos de aprendizagem, partilha de informação/relações formais e informais, aprendizagem individual e ambiente/clima de trabalho.

Com base na investigação empírica efetuada conclui-se também que o caso aqui estudado enfatiza muitas das práticas reconhecidas e analisadas nas dimensões do modelo proposto. Todavia e, tendo presente o novo paradigma da banca no mundo e, particularmente, nos tempos modernos de grande instabilidade e especial provação para o sector financeiro, obrigará a gestão de topo a atenção redobrada a alguns elementos sensíveis e com elevado impacto na performance final do Banco X. Por exemplo, a amizade e o estabelecimento de laços. Nos tempos difíceis que se vivem atualmente, estas dimensões têm um significado extraordinário. Também as lideranças devem ser analisadas com cuidados redobrados e eventualmente avançar para formações e aprendizagem individual de aspetos comportamentais.

Em termos da aprendizagem organizacional, o ambiente/clima deve ser estudado numa perspetiva descentralizada, procurando assim ver a organização globalmente. Já ao nível da aprendizagem individual, referenciando alguns elementos auscultados, conclui-se que é preciso o Banco X avançar para a certificação de funções, escutando periodicamente os seus colaboradores e elevando os seus níveis de aprendizagem.

Nos objetivos académicos deste trabalho estão evidenciados as implicações desta sucinta investigação para a Gestão e, nomeadamente, na temática organizacional. Assim, tal como propalam Fulmer, Gerhart \& Scott (2003), não nos podemos esquecer que as melhores empresas para trabalhar são no plano económico, mas também no humano. Nesta perspetiva, os resultados alcançados devem ajudar à compreensão destas dimensões modernas em ambiente real para o estabelecimento de novos modelos de satisfação no local de trabalho.

Também os modelos de gestão de pessoas nas organizações têm aqui um alfobre de ideias capazes de germinar, em especial, na instituição de 
sistemas de incentivos e de distribuição de resultados ancorados, por exemplo, em climas organizacionais positivos que fomentem $o$ crescimento pessoal. Este estudo abre campo de análise para a importância da partilha de valores organizacionais e a institucionalização de ambientes positivos e de atenção às pessoas e às suas relações. Tendo em atenção o papel de pioneirismo de alguma forma reconhecido às instituições financeiras, especialmente ao nível tecnológico, este trabalho procura também elucidar sobre o que constitui as relações sociais e a aprendizagem organizacional para as equipas de trabalho. Este estudo é relevante do ponto de vista da importância das dimensões estudadas para a perceção moderna do atual status quo de uma instituição financeira e da visão dos seus colaboradores da área comercial em contexto de crise mundial. Assim, o papel das pessoas e das suas inter-relações continua a ser decisivo na institucionalização das organizações modernas, aprendentes e as dimensões verificadas contribuem para o seu aperfeiçoamento.

De acordo com Osório (2009: 264) "a partilha da informação $e$ conhecimento assenta na difusão das vantagens, para a organização $e$ seus trabalhadores, de instrumentos de planeamento tendo em conta os objetivos e as estratégias organizacionais de longo prazo". Assim, as organizações que pretendam manter as suas vantagens competitivas, devem enquadrar estas dimensões enquanto fulcrais para o seu futuro.

Uma limitação do estudo é a falta de transversalidade e a utilização de caso único que não permite a sua generalização. $\mathrm{O}$ uso de várias unidades de análise e o cruzamento de informações com casos de organizações semelhantes possibilitariam conclusões mais sedimentadas.

Por último, o enquadramento da atividade bancária num novo paradigma em construção acelerada, não permite uma sustentação completamente segura das conclusões explanadas, sugerindo-se assim para o futuro, estudos de caso longitudinais que mostrem este tipo de evolução.

Para um reforço das conclusões extraídas, em futuras investigações, sugere-se estudar as inter-relações das dimensões aqui estudadas, descendo a análise ao nível das variáveis que as constituem. Outra linha que merece ser investigada em futuras pesquisas seria também aplicar este tipo de estudo a equipas de gestão de outras organizações, em sectores como os seguros ou serviços tecnológicos, cujos membros individuais da equipa têm idênticas formações de base. 


\section{REFERÊNCIAS}

Aldrich, H., \& Zimmer, C. (1986). Entrepreneurship through social networks. In D. Sexton e R. Smilor (Eds.). The art and science of entrepreneurship. 3-23. Cambridge, MA: Ballinger.

Barney, J. (1991). Firm Resources and Sustained competitive Advantage, Journal of Mnagement, Volume 17, N. ${ }^{\circ} 1$, pp. 99 - 120.

Berliant, M., \& Fujita, M. (2009). Dynamics of knowledge creation and transfer: The two person case. International Journal of Economic Theory, 155-179.

Billett, S. (2004). Workplace participatory practices - conceptualizing workplaces as learning environments, Journal of Workplace Learning, Vol. 16 No. 6, pp. 312-24.

Birley, S. J., (1985). Britain's new enterprise programmes. Journal of Small Business Management, 23(4), 6-12.

Birt, M., Wallis, T. \& Winternitz, G. (2004). Talent retention in a changing workplace: an investigation of variables considered important to South African talent, South African Journal of Business Management, Vol. 35 No. 2, pp. 25-31.

Bonoma, T.V. (1985). "Case Research in Marketing: Opportunities, Problems, and a Process". Journal of Marketing Research, 22(2), 199.

Borgatti, S., \& Cross, R. (2003). A relational view of information seeking and learning in social networks. Management science, Vol. 49, No. 4, pp. 432-445.

Brass, D. J. (1984). Being in the right place: A structural analysis of individual influence in an organization. Administrative Science Quarterly, 29: 518-539.

Brass, D . (1992). Power in organizations: A social network perspective. In G . Moore and J. A. White (Eds.) Research in politics and society . Pp. 295-323 . Greenwich : JAI Press.

Cabrita, M. R. (2009). Capital Intelectual e Desempenho Organizacional. Lisboa: Lidel.

Cascio, W. (1995). Whither industrial and organizational psychology in a changing world of work? American psychologist , 50,11,928-939.

Castells, M. (1999). A sociedade em rede. 2. ed. São Paulo: Paz e Terra.

Charan, R. \& Tichy, N. (1998). Every Business is a Growth Business: How your Company can Prosper Year after Year, Three River Press, New York, NY.

Chollet, B. (2005). The role of the ReD engineer personal network: the case of micro and nanotechnologies sector. $\mathrm{PhD}$ thesis, Pierre Mende`s University, Grenoble. 
Cohen, D. (1998). Toward a Knowledge Context: Report on The First Annual U.C. Berkeley Forum on Knowledge and the Firm'. California Management Review, 40/3: 22-39.

Cohen, W. M. \& Levintal, A. (1990). Absorptive Capacity: A new perspective on learning and innovation. Administrative Science Quarterly, v. 35, p. 128-152.

Coutinho, C. P. (2011). Metodologia de Investigação em Ciências Sociais e Humanas - Teoria e Prática. (L. Almedina, Ed.) (2a ed., p. 343). Coimbra.

Cross, Parker, A., \& Prusak, L. (2000). Knowing What We Know:Supporting knowledge creation and sharing in social networks. Institute for Knowledge Management , 1-34.

Cross, R., \& Sproull, L. (2004). More than an answer: Information relationships for actionable knowledge. Organization Science, 15(4), 446-462.

Crossan, M., Lane, H. \& White, R. (1999). An organizational learning framework: From intuition to institution, Academy of Management Review, 24, 3, 522-537.

Cunha, M. P., Rego, A., \& Cunha A, R. C. (2007). Organizações positivas. Lisboa: Dom Quixote.

Davenport, T. H. (1999). Knowledge Management and the Broad Firm: Strategy, Advantage and Performance. In T. H. Davenport, Knowledge Management Handbook. CRC Press.

Davenport, T. H. (2000). Working Knowledge: How Organizations Manage What They Know. Boston: Harvard Business School.

Davenport, T. H., \& PRUSAK, L. (2000). Working Knowledge: How Organizations Manage What They Know. Harvard: Harvard Business School Press.

Davenport, T.H., \& PrusaK, L. (1998). Working knowledge: how organizations manage what they know. Harvard Business Press.

Davis, G., \& Useem, M. (2001). Top Management, Company. Handbook of Strategy and Management .

Davis, S. (1990). A Excelência na Banca. Lisboa: D.Quixote.

Devlin, J. \& Ennew, C. (1997).Understanding competitive advantage in retail financial services. International Journal of Bank Marketing, 5(3), 73-82.

Dodgson, M. (1993). Organizational learning: a review of some literatures, Organization Studies, Vol. 14 No. 3, pp. 375-94.

Drucker, P. F. (1993). As Fronteiras da Gestão. Lisboa: Presença.

Easterby-Smith, M.; Thorpe, R. \& Jackson, P. (2010). Management Research (3rd edition.). London: Sage. 
Eisenhardt, K. M. (1989). Building theories from case study research. The Academy of Management Review, 14(4), 532-550 .

Estevão, C., \& Franco, M. (2010). O Papel das Parcerias PúblicoPrivadas de Turismo no Desenvolvimento Regional. In S. e. Lourenço, Topicos Avançados de Gestão (p. 373). Covilhã: UBI.

Franco, M., \& Barbeira, M. (2009). Um sistema de gestão do conhecimento como fomentador de redes estratégicas interorganizacionais. Revista Iberoamericana de Estratégia , 8,2,529.

Franco, M., \& Ferreira, T. (2007). Um estudo de caso sobre as organizações que aprendem. Comportamento organizacional $e$ Gestão, 13,2,169-189.

Fulmer, I., Gerharrt, B., \& Scott, K. (2003). Are 100 best better?An empirical investigation of the realtionship between being a "great place to work" and firm performance. Personnel Psychology, 56, 965-993.

Garvin, D. A. (1993). Building a Learning Organization. Harvard Business Review, 71(4), 78-91.

Grant, R. (1991). The resource Based theory of competitive advantage: implications for strategy formulation. California Management Review, 114-135.

Gulati, R. (1998). Alliances and Networks. Strategic Management Journal, 19,293-317.

Håkannson, H. \& Johanson, J. (1988). Formal and Informal Cooperation Strategies in International Industrial Network in Contractor, F. J. and Loranges, P. (Eds.), Cooperative Strategies in International Business, Lexington, Lexington Books, pp. 369-379.

Handy, C. (1995). Trust and the Virtual Organization. HBR May/June, 110.

Hansen, M. T. (1999). The search-transfer problem: The role of weak ties in sharing knowledge across organization subunits. Administrative Science Quarterly, 44: 82-111.

Hedberg, B. (1981). How organizations learn and unlearn. In: Wystrom, P.; Starbuck, W. T. (Eds.). Handbook of Organizational Design. Oxford,UK: Oxford University Press.

Holm U, Johanson J, \& Thilenius P. (1995). Headquarters knowledge of subsidiary network contexts in the multinational corporation. International Studies of Management and Organizations 25(1-2): 97-120.

Imai K., (1989). Evolution of Japan's corporate and industrial networks. in Carlsson B, editor. Industrial dynamics: technological, organizational and structural changes in industries and firms. Boston (MA): Kluwer Academic Publishers, p. 123- 55. 
Inkpen, C.A. \& Crossan, M.M. (1995). Believing is seeing: joint ventures and organization learning, Journal of Management Studies, Vol. 32 No. 5, pp. 595-618.

Inkpen, A.,(1996). Creating knowledge through collaboration, California Management Review, 39 (1) pp. 123-240.

Inkpen, A., \& Tsang. (2005). Social Capital, Networks, and Knowledge transfer. Academy of Management Review , 30, 145-165.

Johannisson, B., (1987). Beyond Process and Structure: Social Exchange Networks, International Studies of Management and Organization 17, 3-23.

Johanson, J., \& Vahlne, J.E. (1990). The mechanism of internationalisation. International Marketing Review, 7(4): 11-24.

Kholer, T., \& Jansen, C. (2010). Communication, social capital and workplace health management as determinants of the innovative climate in German banks. Swiss School of Public Health, 55,561570.

Kilduff, M. (1992). The friendship network as a decisionmaking resource: Dispositional moderators of social influences on organizational choice. Journal of Personality and Social Psychology, 62: 168-180.

Kilduff, M., \& Krackhardt, D. (1994). Bringing the individual back in: A structural analysis of the internal market for reputation in organizations. Academy of Management Journal,37: 87-108.

Kim, D. (1993). The link between individual and organizational learning. Management Review, 37-50.

King, N. \& Anderson, N. (1995). Innovation and Change in Organisations. Routledge, London.

Kogut, B., \& Zander, U. (1995). Knowledge and the speed of the transfer and imitation of organizational capabilities: An empirical test. Organization science , 1,6,76-92.

Kogut, B., \& Zander, U. (1996). What do firms do? Coordination, identity and learning. Organization science , 7-502-518.

Krugman, P. (2009). O Regresso da economia de Depressão e a crise actual. Lisboa: Presença.

Lopes, A. (2008). Fundamentos de uma Epistemologia do Valor das pessoas nas organizações. Lisboa: ISCTE.

Lopes, A., \& Fernandes, A. (2002). Delimitação do conceito de aprendizagem organizacional: sua relação com a aprendizagem individual. Revista Portuguesa e Brasileira de Gestão, 70-75.

Mcdonald, M. L. \& Westphal, J. D. (2003). Getting by with the advice of their friends: CEOs advice networks and firms strategic responses to poor performance. Administrative Science Quarterly, 48, 1-32. 
Mckechnie, S. (1992). Consumer buying behaviour in financial services: An overview. The International Journal of Bank Marketing, 10 (5): 4.

Mehra, A., Kilduff, M., \& Brass, D. J. (2001). The Social Networks of High and Low Self-Monitors: Implications for Workplace Performance. Administrative Science Quarterly, 46(1), 121-146.

Mills, A. J., Wiebe, E. \& Durepos, G. (2010). Encyclopedia of case study research. SAGE Publications.

Mintzberg, H. (2010). Estrutura e dinamica das Organizações. Lisboa: Dom Quixote.

Nahapiet, J., \& Ghoshal, S. (1998). Social capital, intelectual capital, and the organizational advantage. The Academy of Management Review, 23,2-242-266.

Nonaka, I. (1994). A Dynamic Theory of Organizational Knowledge Creation. Organization Science, 14-37.

Nonaka, I., \& TAKEUCHI, H. (1995). The knowledge-creating company : how japonese companies create the dynamics of innovation. New York: Oxford University Press.

Nonaka, I; \& Takeuchi, H. (1997). Criação de Conhecimento na Empresa. 12a. Edição. Rio de Janeiro: Campus,

Osório, J. M. P. (2009). Learning Organizations:as práticas de gestão de recursos humanos. Universidade do Minho.

Popper, M. \& Lipshitz, R. (2000). Organizational learning: mechanisms, culture, and feasibility, Management Learning, Vol. 31 No. 2, pp. 181-96.

Quivy, R. \& Campenhoudt, L. V. . (2008). Manual de Investigação em Ciências Sociais(Gradiva.). Lisboa.

Rank, O., \& Tuschke, A. (2010). Perceived Influence and Friendship as Antecedents of Cooperation in Top Management Teams: A Network Approach. Business Research Journal, Vol. 3, No. 2, November 2010.

Rego, A., Cunha, M., \& Souto, S. (2007). Espiritualidade nas organizações e comprometimento organizacional. RAE-eletrônica, 127.

Rejeb-Khachlouf, N. \& Mezghani, L. (2011). Personal networks and knowledge transfer in inter-organizational networks, Journal of Small Business and Enterprise Development, Vol. 18 No. 2, pp. 278297

Roseira, C., Brito, C., \& Hennenberg, S. C. (2010). Managing interdependencies in supplier networks.Industrial Marketing Management, 39(6), 925-935.

Scandura, T., \& Williams, E. (2000). Research methodology in management: current practices, trends, and implications for future 
research. Academy of Management Journal; Vol. 43 Issue 6, p12481264, 17p.

Senge, P. (1990). The Fifth Discipline: The Art and Practice the Learning Organization.

Senge, P. M. (1996). Leading Learning Organizations. In The Leader of the Future (Goldsmith). Jossey Bass, Inc., a subsidiary of John Wiley e Sons Company,.

Shein, E. (1993). How can organizations learn faster? The challenge of entering the green room. Sloan Management Review, 34,85-92.

Sjoberg, G., Feagin, J. \& Orum, A. (1991). A case for the case study. Chapel Hill, EUA University of North Carolina.

Stevenson, William B. \& Radin, F. (2009). Social Capital and Social Influence on the Board of Directors, Journal of Management Studies, 46 (1): $16-44$.

Strauss, G. (1962). Tactics of Lateral Relationship: The Purchasing Agent. Administrative Science Quarterly, 7(2), 161.

Tichy, N. (1997). The leadership engine: how winning companies build leaders at every level. New York, Harper Business.

Tichy, N. M., \& Bennis, W. G. (2010). Decidir. Lisboa: Actual.

Tsai, W. (2003). Social Structure of "Coopetition" Within a Multiunit Organization. Organization Science, Vol. 13, No. 2, March-April 2002, pp. 179-190.

Unger, H., \& Lorscheider, B. (1996). Groups - a neglected concept in organizational learning, in Growth through Learning, Proceedings of the Annual Conference of the European Consortium for the Learning Organisation (ECLO), Copenhaga, Dinamarca,Maio, pp. 95-105.

Uzzi, B. (1996). The sources and consequences of embeddedness for the economic performance of organizations: The network effect. American Sociological Review, 61(4), 674-698.

Uzzi, B., \& Lancaster, R. (2003). Relational Embeddedness and Learning: The Case of Bank Loan Managers and Their Clients. Management Science, 49(4), 383-399.

Vera, D., \& Crossan, M. (2004). Strategic Leadership and organizational Learning. Academy of Management Review , 29, 2, 222-240.

Visser, C. (2001). Succesvol verandermanagement door appreciative inquiry, ("Succesful change management by appreciative inquiry"), available at: http://m-cc.nl/ succesvolverandermanagement.pdf (accessed October 21, 2013).

Yin, R.K. (2005). Estudo de Caso. São Paulo: Bookman, $3^{\text {a }}$ ed. 
Wernerfelt, B. (1984). A Resource-based View of the Firm, Strategic Management Journal, Vol. 5, pp. 171-180.

Westphal, J. D. (1999). Collaboration in the Boardroom: Behavioral and Performance Consequences of CEO-Board Social Ties, Academy of Management Journal, 42 (1): 7-24. 